\title{
Statin-related Lichenoid Dermatosis: An Uncommon Adverse Reaction to a Common Treatment
}

\author{
Zsófia Vesza, Catarina Pires, Pedro Marques da Silva \\ Hypertension and Dyslipidemia Outpatient Clinic, Medicine 4, Santa Marta's Hospital, CHLC, Lisbon, Portugal
}

Doi: 10.12890/2018_000844-European Journal of Case Reports in Internal Medicine - ㅇ EFIM 2018

\begin{abstract}
Received: $16 / 01 / 2018$
Accepted: 06/02/2018
\end{abstract}

Published: 28/02/2018

How to cite this article: Vesza Z, Pires C, da Silva PM. Statin-related lichenoid dermatosis: an uncommon adverse reaction to a common treatment. EJCRIM 2018;5: doi:10.12890/2018_000844.

\begin{abstract}
Acknowledgements: The authors thank Vera M. Torres, MD (dermatology),and Helena Garcia, MD (anatomical pathology) for diagnostic support. Conflicts of Interests: The Authors declare that there are no competing interests.

This article is licensed under a Commons Attribution Non-Commercial 4.0 License
\end{abstract}

\section{ABSTRACT}

3-Hydroxy-3-methylglutaryl coenzyme A reductase inhibitors (statins) are generally safe and well-tolerated drugs that are extensively used for the primary and secondary prevention of atherosclerotic cardiovascular events. Muscle and liver adverse reactions are the best recognized, whilecutaneous side effects are exceedingly rare. We present the case of a 65-year-old woman with severe hypercholesterolemia, who developed generalized erythematous cutaneous lesions with pruritus, resembling lichen planus, months after starting treatment with simvastatin. The symptoms disappeared on withdrawal of simvastatin and reappeared within 3 months upon rechallenge with rosuvastatin. In addition to describing a rare adverse effect of statins, the authors also discuss the nutraceutical approach to the management of a statinintolerant patient.

\section{LEARNING POINTS}

- Lichenoid drug eruption is an uncommon cutaneous adverse effect of several drugs, with very few cases associated with statins.

- A temporal relationship, dechallenge/rechallenge information, and the lack of confounding factors or alternative explanations support the suggestion of causality.

- Due to the lack of optimized alternative treatment options for statin-intolerant patients, the nutraceutical approach should be considered.

\section{KEYWORDS}

Lichenoid drug eruption, dyslipidemia, statins, nutraceuticals, red yeast rice

\section{INTRODUCTION}

3-Hydroxy-3-methylglutaryl coenzyme A (HMG-CoA) reductase inhibitors (HMGCRi, statins) are frequently prescribed pharmaceutical products. Statins are the mainstay of the management of elevated low-density lipoprotein-cholesterol (LDL-C) levels in patients with high cardiovascular (CV) risk. HMGCRi are well tolerated with a good safety profile $\mathrm{e}^{[1]}$.

The most commonly reported side effects of statins are myotoxicity and hepatotoxicity ${ }^{[1]}$. Statins have also been associated with a number of cutaneous conditions (e.g. eczematous skin rash, cutaneous lupus erythematosus, porphyria cutanea tarda, bullous dermatosis, UV-B phototoxicity, acute generalized exanthematous pustulosis, cheilitis, photosensitivity and dermatomyositis-like syndrome) ${ }^{[2]}$. Lichenoid drug eruption caused by HMGCRi is exceptional[1]. We describe a lichenoid eruption linked to simvastatin and rosuvastatin and a proposed alternative regimen to reduce total cholesterol (TC) and LDL-C in a patient with a severe hypercholesterolemia phenotype. 


\section{CASE REPORT}

A 65-year-old woman presented with symmetric eruption of flat-topped, erythematous papules on the trunk and extremities (forearms and legs), with pruritus. Some of the lesions manifested with a central pustule and marked desquamation, resembling lichen planus. Psoriasiform dermatitis on the palmar surfaces (Fig. 1) and involvement of the lateral border of the tongue was also observed. The patient mentioned similar but milder earlier episodes of cutaneous eruptions in the previous 2 years.

The patient was a former smoker (5 pack/years) with a body mass index (BMI) of $26.6 \mathrm{~kg} / \mathrm{m}^{2}$ and a clinical history of hypercholesterolemia, pulmonary emphysema, autoimmune thyroiditis (currently euthyroid), and an allergy to sulphonamide antibiotics. She had been taking simvastatin $20 \mathrm{mg}$ /day for approximately 2 years with an apparently good response to the treatment, and denied concurrent medication or initiation of any new cosmetics or detergents. The patient's dermatological symptoms resolved when simvastatin was suspended.

Hypercholesterolemia treatment was resumed later, with rosuvastatin $10 \mathrm{mg}$ daily. However, identical generalized cutaneous lesions reappeared after 3 months. Skin biopsies revealed lichenoid lymphohistiocytic infiltrate in the papillary dermis, focal parakeratosis, necrotic keratinocytes and exocytosis of lymphoid cells into the epidermis (Figs. 2 and 3). A skin prick test showed a positive reaction to fragrances. Cessation of HMGCRi with concomitant PUVA (psoralen and ultraviolet A radiation) therapy and oral corticosteroid treatment resulted in complete remission of cutaneous lesions 6 months later.



Figure 1. Psoriasiform dermatitis on palms of the hands with marked desquamation

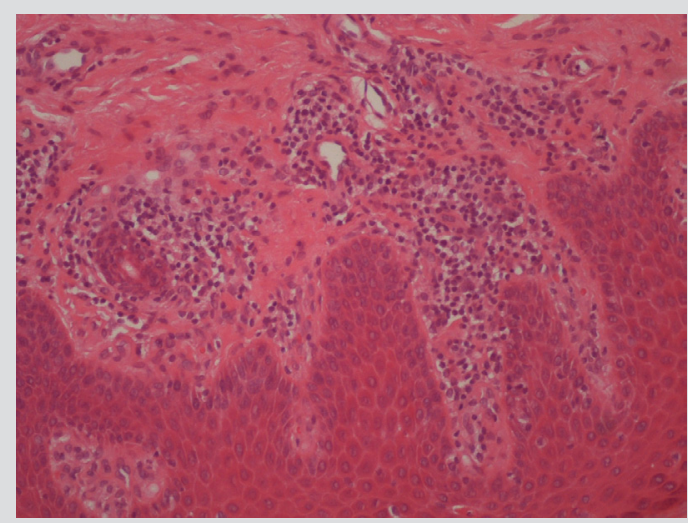

Figure 2. Histologic analysis of the skin biopsies showing lymphohistiocytic infiltrate in the papillary dermis in a lichenoid distribution and exocytosis of lymphoid cells into the epidermis

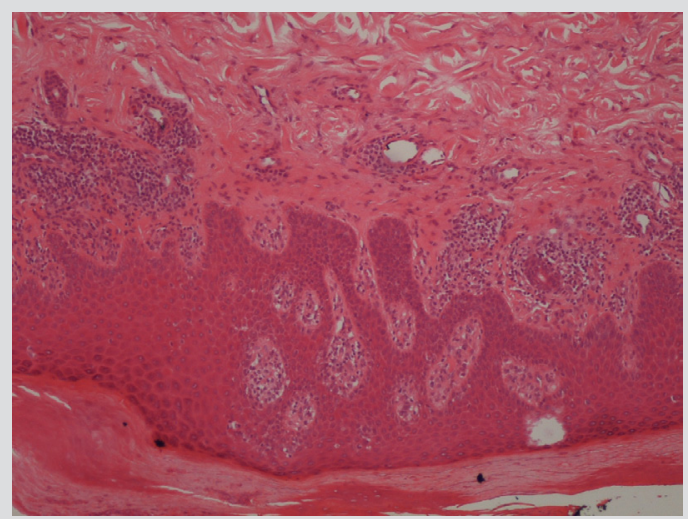

Figure 3. Histologic analysis of the skin biopsies showing lymphohistiocytic infiltrate in the papillary dermis in a lichenoid distribution and exocytosis of lymphoid cells into the epidermis (greater magnification) 


\section{of Case Reports in \\ Internal Medicine}

However, with the disruption of lipid-lowering treatment the patient's lipid profile deteriorated, showing a fasting LDL-C of $208 \mathrm{mg} / \mathrm{dl}$, a high-density lipoprotein-cholesterol (HDL-C) of $65 \mathrm{mg} / \mathrm{dl}$, an apolipoprotein B (ApoB) of $153 \mathrm{mg} / \mathrm{dl}$, triglycerides (TG) of $136 \mathrm{mg} / \mathrm{dl}$, and a lipoprotein (a) of $9 \mathrm{mg} / \mathrm{dl}$ (Table 1). In addition to lifestyle interventions to reduce TC and LDL-C levels, ezetimibe $10 \mathrm{mg} / \mathrm{day}$ was introduced, along with nutraceutical treatment which consisted of a combination of red yeast extract (200 mg, corresponding to $3 \mathrm{mg}$ of monacolin), policosanol (10 mg) and berberine ( $500 \mathrm{mg}$ ), in addition to folic acid $(0.2 \mathrm{mg}$ ), astaxanthin ( $0.5 \mathrm{mg})$ and coenzyme Q10 (2 mg). After $4 \mathrm{months}$, the lipid profile had steadily improved (Table 1). After 16 months, the patient's laboratory tests revealed LDL-C $105 \mathrm{mg} / \mathrm{dl}$, HDL-C $65 \mathrm{mg} / \mathrm{dl}$, ApoB $98 \mathrm{mg} / \mathrm{dl}$ and TG $90 \mathrm{mg} / \mathrm{dl}$. She remained asymptomatic, with no evidence of any further cutaneous lesions.

\begin{tabular}{|c|c|c|c|c|c|c|c|c|c|c|c|c|c|c|c|}
\hline Year & 2009 & 2010 & 2011 & 2012 & \multicolumn{3}{|c|}{2013} & \multicolumn{3}{|c|}{2014} & \multicolumn{2}{|c|}{2015} & 2016 & \multicolumn{2}{|c|}{2017} \\
\hline Month & 12 & 3 & 3 & 11 & 3 & 8 & 11 & 2 & 4 & 8 & 2 & 8 & 6 & 1 & 11 \\
\hline $\mathrm{TC}(\mathrm{mg} / \mathrm{dl})$ & 326 & $263^{*}$ & $258^{*}$ & 276 & $343 t$ & 293 & 297 & 300 & 287 & $238 \neq$ & $193 \ddagger$ & $188 \neq$ & & 187‡ & 197‡ \\
\hline $\mathrm{HDL}-\mathrm{C}(\mathrm{mg} / \mathrm{dl})$ & 75 & $70^{*}$ & $70^{*}$ & 63 & $80+$ & 67 & 67 & 72 & 65 & $69 \neq$ & $70 \neq$ & $65 \ddagger$ & & $55 \ddagger$ & $57 \ddagger$ \\
\hline $\operatorname{LDL}-\mathrm{C}(\mathrm{mg} / \mathrm{dl})$ & 230 & $166^{*}$ & $171^{*}$ & 184 & $239+$ & 208 & 224 & 215 & 208 & $145 \ddagger$ & $105 \ddagger$ & $105 \ddagger$ & $112 \ddagger$ & $112 \ddagger$ & $116 \neq$ \\
\hline TG (mg/dl) & 101 & $135^{*}$ & $88^{*}$ & 144 & $124 t$ & 90 & 150 & 144 & 136 & $119 \ddagger$ & $89 \neq$ & $90 \neq$ & $63 \ddagger$ & $103 \ddagger$ & $123 \neq$ \\
\hline$A p o B(m g / d l)$ & & & & & & 158 & 146 & 143 & 153 & $110 \ddagger$ & & $98 \neq$ & & $95 \ddagger$ & $101 \neq$ \\
\hline $\operatorname{Lp}(a)(m g / d l)$ & & & & & & 9 & & & 9 & & & $8 \neq$ & & & \\
\hline
\end{tabular}

Table 1. Lipid profile evolution of the patient

*Simvastatin $20 \mathrm{mg} /$ day.

†Rechallenge with rosuvastatin.

\#Ezetimibe $10 \mathrm{mg} /$ day + Armolipid ${ }^{\circledast}$ Plus.

ApoB: apolipoprotein B; HDL-C: high-density lipoprotein-cholesterol; LDL-C: low-density lipoprotein-cholesterol; Lp(a) a lipoprotein (a); TC: total cholesterol; TG: triglycerides.

\section{DISCUSSION}

Lichenoid drug eruption is most frequently related to $\beta$-blockers, captopril, penicillamine, hydrochlorothiazide, antimalarials, furosemide, quinidine, non-steroidal anti-inflammatory drugs, tetracycline, quinacrine, gold, sulfonylureas, hydroxyurea and methyldopa ${ }^{[3]}$. Although its pathogenetic mechanism is incompletely understood, cell-mediated autoimmune reactions against basal layer keratinocytes are thought to be involved ${ }^{[3]}$.

The latent period between the initiation of the offending drug and the appearance of the cutaneous lesions varies from months to a year or more, and depends upon the type of drug, dosage and concurrent medications. Establishing a relationship between a suspect agent and the adverse reaction is also complicated by multiple drug use in most patients. The diagnosis is confirmed by the characteristic clinical presentation and histopathological findings, and can be distinguished from idiopathic lichen planus following a washout period by observing the reappearance of the same symptoms after re-administration of the suspect drug (dechallenge/rechallenge test) ${ }^{[4]}$. Treatment includes elimination of the offending drug, and administration of oral and topical glucocorticoids. The time to resolution of symptoms after removal of the causative agent ranges from a couple of weeks to a year ${ }^{[3]}$.

To the best of our knowledge, there are only a limited number of case reports of lichenoid drug eruption associated with statins ${ }^{[1]}$. In our case, HMGCRi was highly likely to be the offending drug because rechallenge with another statin also caused lichenoid eruption. The temporal relationship also supported this hypothesis. Drug interactions that could potentiate the adverse effects were ruled out as the patient was not taking any concomitant medications.

Ezetimibe is indicated as first-line therapy in the case of proven intolerance to statins ${ }^{[5]}$. Bile acid sequestrants (currently unavailable in Portugal) and proprotein convertase subtilisin/kexin 9 (PCSK) inhibitors may additionally be considered. In recent years, nutraceuticals have emerged as possible adjuvant treatment for lowering TC and LDL-C in hypercholesterolemic patients intolerant to statins ${ }^{[6]}$.

The combination of ezetimibe with a low dosage of nutraceuticals with different lipid-lowering properties and mechanisms of action can result in synergetic effects, affecting the bowel absorption of lipids (ezetimibe), increasing the hepatic uptake of cholesterol (ezetimibe, berberine), inducing LDL-C excretion (berberine), inhibiting HMGCR and limiting the hepatic synthesis of cholesterol (monacolins, 




policosanols) ${ }^{[6]}$. Nutraceuticals and ezetimibe have shown consistent incremental lipid-lowering efficacy in statin-intolerant dyslipidemic subjects with no apparent undesirable effects and no safety concerns, but data are limited and further studies are necessary ${ }^{[6]}$. Regular monitoring for adverse reactions and lipid changes after starting alternative treatments should be carried out, especially in statin-intolerant patients.

\section{REFERENCES}

1. Golomb BA, Evans MA. Statin adverse effects: a review of the literature and evidence for a mitochondrial mechanism. Am J Cardiovasc Drugs 2008;8:373-418.

2. Jowkar F, Namazi MR. Statins in dermatology. Int J Dermatol 2010;49:1235-1243.

3. Jain S. Dermatology. Illustrated study guide and comprehensive board review. 2nd ed. Springer: Switzerland, 2017, pp. $106-109$.

4. Medicines and Healthcare products Regulatory Agency (MHRA). Good pharmacovigilance practice guide. RPS Publishing: Cambridge, 2009, pp. 29-48.

5. Barrios V, Escobar C, Cicero AF, Burke D, Fasching P, Banach M, Bruckert E. A nutraceutical approach (Armolipid Plus) to reduce total and LDL cholesterol in individuals with mild to moderate dyslipidemia: review of the clinical evidence. Atheroscler Suppl 2017;24:1-15.

6. Burke FM. Red yeast rice for the treatment of dyslipidemia. Curr Atheroscler Rep 2015;17:495. 